Article

\title{
Research on Transmission Network Expansion Planning Considering Splitting Control
}

\author{
Fei Tang ${ }^{\circledR}$, Chufei Xiao *, Xin Gao, Yifan Zhang, Nianchun Du and Benxi Hu \\ School of Electrical Engineering and Automation, Wuhan University, Wuhan 430072, China; \\ tangfei@whu.edu.cn (F.T.); 2019202070018@whu.edu.cn (X.G.); 2019202070074@whu.edu.cn (Y.Z.); \\ 2019202070077@whu.edu.cn (N.D.); 2019202070073@whu.edu.cn (B.H.) \\ * Correspondence: xiaochufei@whu.edu.cn; Tel.: +86-1347-702-8442
}

Received: 7 January 2020; Accepted: 24 February 2020; Published: 27 February 2020

check for updates

\begin{abstract}
A robust and reliable grid is one of the core elements for power network planning. Specifically, splitting is an effective way for power grid out-of-step oscillation. Since the cross-section of system out-of-step is mostly found on the weak connection lines, reducing the number of those lines can be conducive to the system partition, save the finding time of the optimal splitting cross-section, and improve the performance of the splitting control. This paper proposed an enhanced method based on slow coherence theory for weak connection lines' identification and monitoring. The ratio of the number of weak connection lines to the number of all the lines, called weak connection coefficient, is considered as a crucial factor. A bi-level programming model, which perceives the minimum connection coefficient as the optimization goal, is built for the transmission network. Additionally, a fused algorithm, consisting of Boruvka algorithm and particle swarm optimization with adaptive mutation and inertia weight, is employed to solve the proposed method in the instances of an 18-node IEEE Graver system and a practical power grid in East China. Simulation results in PSD-BPA are conducted to verify the effectiveness of the weak connection monitoring method and transmission network planning model.
\end{abstract}

Keywords: power system; power network planning; weak connection; splitting control

\section{Introduction}

A proper power network structure is the prerequisite for the safe operation of the power system and a reliable smart grid [1,2]. With the advent of the ultimate high voltage power transmission era in China, the power system is expanding to an unprecedented scale. In this way, the intricated operation characteristics and cross-section out-of-step oscillations have placed challenges for the interconnected power grid [3,4]. For example, the 2018 southern Brazil and the 2019 Venezuela blackout caused industry stagnation and thus, innumerable economic loss [5,6]. Splitting is the last defense line of the power grid to avoid voltage collapse as it can effectively eliminate the out-of-step oscillation $[7,8]$. Therefore, in the early transmission planning stage, if the latent oscillation pattern caused by large disturbances and corresponding splitting control procedures could be recognized and researched, possibly, some of the major disasters could be avoided.

Approximately, power network planning has been clustered into three categories:

1. The requirement for reliability is added to the optimization problem as constraints, usually including the overload constraints under $\mathrm{N}, \mathrm{N}-1$, even $\mathrm{N}-2$, and $\mathrm{N}-\mathrm{K}$ contingency-constrained grid operations $[9,10]$. However, most of the time, the economic operation of the power grid is the main optimization goal, which leads to the postponement of the safety analysis and complexity of computations. 
2. The requirement for reliability is converted into the economic cost in the objective function to obtain the least expensive plan. The cut-off load when the branch is overloaded is quantified as the load shedding compensation cost, and the expected amount of system power shortage is quantified as the power shortage cost. According to the load shedding compensation cost, and the power shortage cost, the level of power supply reliability is reflected [11,12].

3. From the perspective of both reliability and economics, the planning is attained through multiobject programming. To be specific, it can be divided into two kinds. First, the benchmark could be the weighted sum of the requirement for reliability and economics. The weight could be determined by the theory of rough set, fuzzy set, credibility, and so forth [13,14]. Second, hierarchical planning theory could be used to construct a multiobject optimization model, thus, to find the optimal global solution by integrating modern heuristic algorithms and through iterations $[15,16]$.

The planning approaches above tend to exclusively consider the role of the first two defense lines in system protection, and only a few of them take the role of the third defense line into account. After a large disturbance, the grid with complicated operation modes might yield relatively complex oscillation patterns. Simple relay protection actions and load cutting may not be able to cope with the above complex scenarios. To fill in the research gap, this paper proposes a transmission network planning Figure considering splitting control.

One of the key points of splitting control is the selection of splitting sections. When out-of-step oscillations occur in power systems, the splitting sections are mostly concentrated near certain specific lines, which are defined as weak connection lines of the system [17,18]. Accurately identifying and screening the weak connection lines of the system can narrow the monitoring scope during the line monitoring, provide important reference for the selection of the splitting section during the splitting control, reduce the search time, enable the splitting devices installed in the system to better cooperate with the splitting control, and further enhance the effectiveness of the implementation of the splitting control. Starting from the slow coherence theory, through the decomposition and aggregation of the system area, the strong correlation between the weak connection lines and the slow mode eigenvalues of the system is derived, and the weak correlation between the lines inside the coherence cluster and the slow mode eigenvalues is derived [17]. Based on this conclusion, the weak connection lines are screened according to the sensitivity by solving the sensitivity of the lines to the slow mode eigenvalues. This method does not need to divide the node mode of the system, and the calculation is simpler, but there is a problem that the weak connection lines in the system cannot be accurately screened, which depends on the judgment of the network topology manually. Based on previous studies, combined with the modified cosine similarity factor clustering method and graph theory, this paper proposes an improved system of weak connection identification and screening method, which can automatically and accurately screen weak connection lines without artificial experience $[19,20]$.

Based on the multi-timescale slow coherence theory, this paper first analyzes the feasibility of splitting the weak connection lines in the system and proposes an improved method to identify and screen the weak connection lines in the system and a bi-level planning model of transmission network considering splitting control. Finally, the hybrid algorithm, consisting of the topology connection repair strategy based on Boruvka algorithm and PSO with adaptive mutation and inertia weight, is applied to solve the model in IEEE 18 node system and a real power grid in East China. The resulting planning Figure is verified in PSD-BPA, which proves the feasibility and effectiveness of this method.

\section{Weak Connection Lines}

\subsection{Slow Coherency Theory for Power System}

In the actual operation process of power grid, a large-scale interconnected power grid will appear in the phenomenon of coherency and cluster instability after a specific disturbance, where coherency refers to the group of generator sets with the same or similar movement change trend of generator rotor after the disturbance, and cluster instability denotes loss of synchronism between this group of 
units and other clusters [21]. The slow coherence theory has two-time scale properties, which can be divided into slow mode and fast mode. A large number of actual operation and simulation examples show that the slow mode between the grouped generators can reflect the inherent unit oscillation mode of the system [22]. Through the analysis of the slow mode eigenvalue of the state matrix of the linear system, the coherency identification of the generating units in the system can be effectively carried out.

According to the definition of coherence in slow coherence theory [23-26], for a linear system $\ddot{x}=$ $A x$, let $x(t)$ be the state variable of the system. If $\lambda_{\mathrm{i}}$ is the eigenvalue of $A$, then the eigenvalue of the second-order system is $\pm \sigma_{\mathrm{i}}$, as shown in Equation (1):

$$
\sigma_{i}=\sqrt{\lambda_{i}}
$$

Let $\mu_{\mathrm{m}}=\left\{\lambda_{1}, \lambda_{2}, \ldots, \lambda_{\mathrm{m}}\right\}$ be a set of eigenvalues of matrix $A$. If the two-state variables $x_{\mathrm{i}}(t)$ and $\mathrm{x}_{\mathrm{j}}(t)$ in the system state variables $x(t)$ satisfy $x_{\mathrm{i}}(t)-x_{\mathrm{j}}(t)=z_{\mathrm{ij}}(t)$, and $z_{\mathrm{ij}}(t)$ does not contain the corresponding eigenvalues $\left\{ \pm \sigma_{1}, \pm \sigma_{2}\right.$ for any one of $\left.\pm \sigma_{\mathrm{m}}\right\}, \mathrm{x}_{\mathbf{i}}(t)$, and $\mathrm{x}_{\mathbf{j}}(t)$ are defined as coherent variables to the eigenvalue group $\mu_{\mathrm{m}}$. In this regard, if the state variables of matrix $A$ are divided into groups, any two-state variables in the group are all coherent to the eigenvalue group $\mu_{\mathrm{m}}$, and $\mu_{\mathrm{m}}=\left\{\lambda_{1}, \lambda_{2}, \ldots, \lambda_{\mathrm{m}}\right\}$ is a group of eigenvalues with the lowest absolute value, then the system is deemed to be slow coherent to the eigenvalue group $\mu_{\mathrm{m}}$. Precisely, $\lambda_{1}, \lambda_{2}, \ldots, \lambda_{\mathrm{m}}$ is called the slow mode eigenvalue of the system.

\subsection{Feasibility Analysis for Splitting the Weak Connection Lines}

When the power system is unstable, the core problem of the feasibility of splitting the weak connection lines includes: first, whether the splitting at the weak connection can effectively isolate the fault; second, whether the weak connection lines obtained in the steady-state are still applicable after the system is disturbed.

When the system is disturbed, there are two kinds of transient instability; one is a single machine out-of-step, the other is asynchronous generator group out of stability [27], because the main goal of the splitting control is to physically isolate the asynchronous cluster after the system is disturbed, reconstruct the power balance as much as possible, and prevent the further expansion of the fault. Hence, this paper mainly considers the asynchronous generator group instability phenomenon that may occur in the system, because the weak connection lines are distributed among the slow mode areas, the cut-off of weak connection lines can effectively isolate each area and avoid fault propagation.

The topological structure, operation mode, unit parameters, and disturbance of the power grid all affect the coherency grouping of generators to varying degrees. Among them, the topological structure of the power grid, the power flow distribution in steady-state, and unit parameters determine the internal oscillation mode of the system, because the disturbance of the system only sparks one or several of the inherent oscillation modes of the excitation system. The oscillation modes and the weak connection lines between the modes can be independently determined without the fault location and the type of fault, which lays the foundation of this study.

There are two kinds of system faults, one is an erasable fault, and the other is permanent fault [20]. When the system has a fault that can be cleared, because the topology of the system has not changed, the weak connection lines obtained in the steady-state can still be used when the system is disturbed; whereas when the system has a permanent fault, the part of the fault lines needs to be cut off. However, since only part of the lines inside a slow mode area has been changed, other slow mode areas have not been significantly affected, so in other regions, splitting can still be carried out at the weak connection lines obtained in the steady-state to isolate the fault area in time to prevent more complex changes in the oscillation mode.

In Figure 1 , it is assumed that the system can be divided into three slow mode areas $R_{1}, R_{2}$, and $R_{3}$. The weak connection lines between slow modes are $L_{1}, L_{2}$, and $L_{3}$. The gray circle represents the generator node, and the white circle represents the load node. When the fault location and type change, it may cause grouping of $R_{1}, R_{2}$, and $R_{3} ; R_{1}$ and $R_{2}, R_{3} ;$ or $R_{1}, R_{2}$, and $R_{3}$, respectively. However, 
no matter how the fault mode is changed, the weak connection lines $L_{1}, L_{2}$, and $L_{3}$ can be used as the pre-selected lines for splitting to isolate the fault effectively. To sum up, the weak connection lines of the system can be used as the splitting lines to be selected to avoid the spread of blackout caused by the migration of the oscillation center between regions.

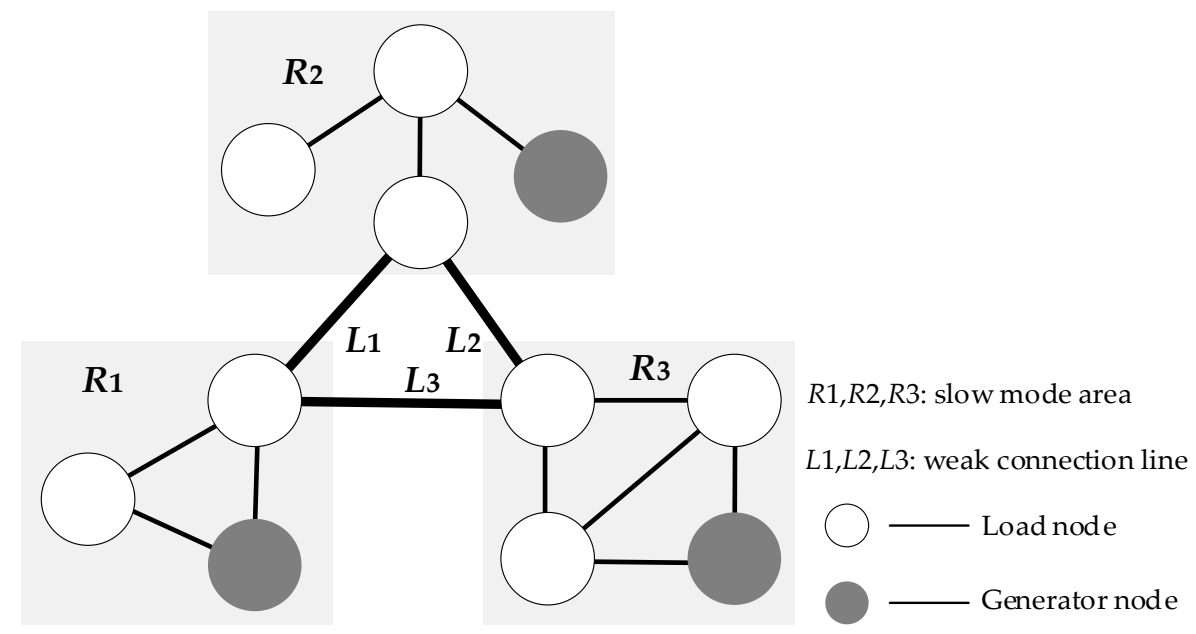

Figure 1. Schematic diagram of the slow mode area.

\subsection{Identification and Screening of Weak Connection Lines in the System}

Identification and screening of weak connection lines is the key to consider splitting control in the initial stage of grid planning. The specific steps of improving identification and screening of weak connection links are as follows.

\subsubsection{Solving State Matrix A}

The slow coherence theory adopts a linearized power system model for coherency clustering [28]. For this reason, a classical second-order synchronous generator model ignoring the salient pole effect and transient effect of the excitation winding is adopted in this paper. The rotor motion equation of the generator can be expressed as Equation (2):

$$
\left\{\begin{array}{l}
\frac{d \delta_{i}}{d t}=\Omega\left(\omega_{i}-1\right) \\
2 H_{i} \frac{d \omega_{i}}{d t}=-D_{i}\left(\omega_{i}-1\right)+\left(P_{m i}-P_{e i}\right)
\end{array}\right.
$$

The mechanical input power $P_{\mathrm{mi}}$ of those mentioned above in the classical second-order synchronous generator model is usually assumed to be a constant, and its electromagnetic output power $P_{\text {ei }}$ can be expressed as Equation (3):

$$
P_{e i}=\sum_{\substack{j=1 \\ j \neq i}}^{n} E_{i} E_{j}\left(B_{i j} \sin \delta_{i j}+G_{i j} \cos \delta_{i j}\right)+E_{i}^{2} G_{i j}
$$

Neglecting the influence of damping winding of the synchronous motor, Equation (4) can be obtained by linearizing Equation (3) near the steady value of generator power $P_{\mathrm{ei} 0}$ and the steady value of power angle $\delta_{\mathrm{i} 0}$.

$$
\ddot{x}=-(1 / 2) \Omega H^{-1} K x \triangleq A x
$$


The calculation formula of system state matrix $K$ is as follows (Equation (5)):

$$
\left\{\begin{array}{c}
K_{i j}=\frac{\partial P_{e i}}{\partial \delta_{j}}=E_{i} E_{j}\left[-G_{i j} \sin \delta_{i j}+B_{i j} \cos \delta_{i j}\right] \\
K_{i i}=-\sum_{\substack{j=1 \\
j \neq i}}^{n} K_{i j}
\end{array}\right.
$$

\subsubsection{Screening System Slow Mode Eigenvalues}

All eigenvalues of the state matrix $A$ can be calculated according to Equation (4); each eigenvalue represents a mode state of the system. Additionally, the absolute values of the eigenvalues of the matrix $A$ are sorted from small to large: $\lambda_{1}, \lambda_{2}, \ldots, \lambda_{\mathrm{n}}$. It can be seen from the theory of slow coherence that the more the value of $\lambda_{\mathrm{m}} / \lambda_{\mathrm{m}+1}$ is, the more obvious the regionality of the system is. The number of slow mode eigenvalues and slow mode eigenvalue group $\mu_{\mathrm{m}}=\left\{\lambda_{1}, \lambda_{2}, \ldots, \lambda_{\mathrm{m}}\right\}$ can be determined by this principle. The specific formula is as follows (Equation (6)):

$$
\lambda_{m} / \lambda_{m+1}=\min _{i=2,3, \ldots, n} \lambda_{i} / \lambda_{i+1}
$$

\subsubsection{Generator Coherence Clustering based on the Modified Cosine Similarity Factor}

As the traditional clustering matrix cannot accurately group the generators, this paper uses the clustering method based on the modified cosine similarity factor to measure the correlation between units by calculating the cosine value of the angle between the eigenvectors of each slow mode eigenvalue and introduces the eigenvector of the slow mode eigenvalue $\left\{v_{1}, \ldots, v_{2}, v_{\mathrm{m}}\right\}$ average value $v_{\mathrm{mo}}$ in $m$ dimension to balance the difference of vector-matrix in each dimension value, and more clearly reflects the coherency between units in $m$ dimension matrix. The specific formula for calculating the clustering matrix $L_{\mathrm{ij}}$ is as follows (Equation (7)):

$$
L_{i j}=\frac{\sum\left(v_{i m}-v_{m o}\right)\left(v_{m k}-v_{m o}\right)}{\sqrt{\sum\left(v_{i m}-v_{m o}\right)^{2} \sum\left(v_{j m}-v_{m o}\right)^{2}}}
$$

2.3.4. Calculate the Sensitivity of the Slow Mode Eigenvalue Group to Each Line

By calculating the sensitivity $\left\{D_{\lambda_{1}}^{l}, \ldots, D_{\lambda_{2}}^{l}, D_{\lambda_{m}}^{l}\right\}$ of each slow mode eigenvalue to the line $l$ to measure the strength of the line, the formula is as follows (Equation (8)):

$$
D_{\lambda_{i}}^{l}=-\frac{\partial \lambda_{i}}{\partial B}+\frac{R}{X} \times \frac{\partial \lambda_{i}}{\partial G}
$$

The sensitivity of slow mode eigenvalue group to the line $l$ is (Equation (9)):

$$
D_{\mu_{m}}^{l}=\sum_{i=1}^{m} D_{\lambda_{i}}^{l}
$$

\subsubsection{Screening Weak Connection Lines of the System}

Since the greater the sensitivity of the line in the system means that the line is weaker than other lines, it can be judged whether the line is suitable for splitting by taking the sensitivity of the line as the weight. The sensitivity of the line obtained by Equation (9) is normalized according to the maximum sensitivity of the line in the current system.

According to the clustering matrix $L \mathrm{ij}$, the results of the generator coherence group are obtained, and the number of slow mode regions is determined. First, disconnect all lines, take the generator node with the maximum output in each area as the initial node of the area, only one node can be added 
to one area in each screening, connect the line with the least sensitivity connected to the area, and add the connection node of the line to the area. Then start the next filter. In the process of screening, when a node is connected with two different slow mode areas, it can judge which area to join according to the sensitivity of the connecting lines between the node and the two areas, and disconnect the lines connected with the other slow mode area.

When the screening is completed, the lines between slow mode areas in the system are weak connection lines, and the number $k$ of weak connection lines is the sum of these lines.

\section{Double Level Programming Optimization Model of Transmission Network}

\subsection{Two-Level Programming Model of Transmission Network Considering Splitting Control}

The upper objective function of the two-level planning model of transmission network considering splitting control proposed in this paper includes three parts: line construction cost, network loss cost, and load shedding cost. The lower objective function takes the minimum weak connection coefficient of the system as the optimization objective, and the mathematical model established is as follows (Equations (10) and (11)):

$$
\begin{gathered}
F_{1}=\min \left(C_{1}+C_{2}+C_{3}\right) \\
F_{2}=\min \left(C_{0}\right)+U
\end{gathered}
$$

\subsection{Objective Function}

\subsubsection{Weak Connection Coefficient of the System}

As the comparison of the number of weak connection lines in different planning Figures is meaningless after leaving the planning grid, this paper defines the ratio of the number of weak connection lines in the system to the total number of lines in the system as the weak connection coefficient of the system, to solve the numerical difference in direct comparison of the number of weak connection lines in different planning grids. According to Section 3.1, the number of weak connection lines in the system can be calculated by the improved method of selecting weak connection lines in the system, and the calculation formula of the weak connection coefficient of the system can be obtained as follows (Equation (12)):

$$
C_{0}=k / \sum l
$$

The constraint to be met in the calculation process is:

Generator coherence constraint:

Generators of the same slow mode cluster should be distributed in the same area when meeting the topological connectivity.

\subsubsection{Line Construction Cost}

The line construction cost refers to the equivalent annual value of the construction cost of the new construction lines required by the system planning. The calculation formula is as follows (Equation (13)):

$$
C_{1}=\frac{a(a+1)^{r}}{(a+1)^{r}-1} \sum_{i \in m_{l}} k_{1} l_{i} L_{i}
$$

\subsubsection{Network Loss Cost}

In this paper, the network loss cost refers to the active loss cost generated by the annual operation of the system. The calculation formula is as follows (Equation (14)):

$$
C_{2}=\sum_{i \in m_{l}} \tau_{i} k_{2} r_{i} P_{i}^{2} / U_{i}^{2}
$$




\subsubsection{Load shedding cost}

To avoid the lack of close connection between nodes in each area and ensure the reliability of power supply, the optimization objective is to minimize the cost of load shedding under $\mathrm{N}$ and $\mathrm{N}-1$ conditions. The calculation formula is as follows (Equation (15)):

$$
C_{3}=\sum_{i \in m_{l}} k_{3}\left(P_{L}^{N}+P_{L}^{N-1}\right)
$$

\subsection{Other Constraints}

Other constraints are:

(a) Network connectivity constraint: The planned grid structure needs to meet the most basic network topology connectivity.

(b) AC power flow constraint (Equation (16)):

$$
P_{i}=V_{i} V_{j}\left(G_{i j} \cos \theta_{i j}+B_{i j} \sin \theta_{i j}\right)-V_{i}^{2} G_{i j}
$$

(c) Node power balance constraints (Equation (17)):

$$
\begin{aligned}
& P_{G, i}-P_{L, i}=V_{i} \sum_{j=1}^{n} V_{j}\left(G_{i j} \cos \theta_{i j}+B_{i j} \sin \theta_{i j}\right) \\
& Q_{G, i}-Q_{L, i}=V_{i} \sum_{j=1}^{n} V_{j}\left(B_{i j} \cos \theta_{i j}-G_{i j} \sin \theta_{i j}\right)
\end{aligned}
$$

(d) Transmission capacity constraint (Equation (18)):

$$
\left|P_{i j}\right| \leq P_{i j \max }
$$

(e) Generator node output constraint (Equation (19)):

$$
P_{G_{\min }} \leq P_{G} \leq P_{G_{\max }}
$$

(f) A maximum number of line construction constraint (Equation (20)):

$$
0 \leq l_{i} \leq l_{\text {imax }}
$$

\section{Solution Algorithm and Process}

\subsection{Particle Swarm Optimization Algorithm with Adaptive Mutation and Inertia Weight}

Particle swarm optimization (PSO) simulates the process of birds swarming in search of food. Each particle is equivalent to a bird with only position and speed. The fitness value of the objective function is the food the birds' swarm seeks. Each iteration adjusts the speed and position of particles according to the optimal value of individual fitness value and population fitness value. To avoid premature convergence of PSO, adaptive mutation operation, and nonlinear dynamic methods are introduced in this paper [29,30].

\subsubsection{Particle Speed and Position Update}

The formula of particle velocity and position is (Equations (21) and (22)):

$$
v_{i d}^{t+1}=\omega v_{i d}^{t}+c_{1} r_{1}\left(p_{i d}^{t}-x_{i d}^{t}\right)+c_{2} r_{2}\left(p_{g d}^{t}-x_{i d}^{t}\right)
$$




$$
x_{i d}^{t+1}=x_{i d}^{t}+v_{i d}^{t+1}
$$

\subsubsection{Adaptive Mutation Operation}

To solve the problem of premature convergence of particle swarm optimization (PSO), an adaptive mutation operation is introduced into PSO, and a mutation threshold is set in the iteration process so that some particle variables can change with a certain probability. Mutation operation expands the diversity of particle populations and improves the possibility of finding the optimal value (Equation (23).

$$
x_{i d}^{k}= \begin{cases}x_{i d}^{k}(1+0.5 \eta) & \mu \geq p \\ x_{i d}^{k} & \mu<p\end{cases}
$$

\subsubsection{Adaptive Adjustment of Inertia Weight}

The inertia weight of particle swarm optimization can balance the global optimization ability and local optimization ability of the algorithm. To avoid the algorithm falling into local optimization as much as possible, the nonlinear dynamic method can be used to adjust the inertia weight in the iteration. The formula is as follows (Equation (24):

$$
\omega_{t}=\omega_{\text {end }}+\left(\omega_{\text {start }}-\omega_{\text {end }}\right) \exp \left(-k \times\left(t / t_{\max }\right)^{2}\right)
$$

\subsection{Based on Topological Connectivity Repair Strategy of Boruvka Algorithm}

In the process of iteration, particle swarm optimization may generate a disconnected planning Figure, which is excluded by the penalty function in traditional transmission network planning. In this paper, based on the Boruvka algorithm, a topology connected repair strategy is adopted. All nodes and edges are traversed based on the topology of the unconnected planning Figure, and the smallest edge connecting the independent connected regions is found until only one connected region is left in the network. This method can effectively transform the disconnected Figure into the connected Figure and calculate more planning Figures within a certain number of iterations, to improve the calculation efficiency of the algorithm.

\subsection{Solution Flow}

The basic idea of bi-level optimization is first to solve the upper objective function and get a set of grid planning. The optimal solution set, then the lower objective function is calculated based on the solution set of the upper objective function, and then the fitness value of the lower objective function is fed back to the upper objective function in the form of penalty function, repeatedly iterating, from which the optimal solution is selected as the final solution of the planning, reflecting the interaction of the upper and lower models. According to the characteristics of the two-level planning model of the transmission network, this paper uses a hybrid algorithm based on the Boruvka algorithm topology connectivity repair strategy and the particle swarm optimization algorithm of adaptive mutation and inertia weight. The upper and lower layers cooperate. The solution flow is shown in Figure 2. The specific steps are as follows:

(i) According to the initial data of the line, planning Figures are generated, and the initial parameters of the algorithm are initialized.

(ii) Adjust inertia weight and mutation operation adaptively, update particle position, velocity, individual extremum, and population extremum.

(iii) Judge whether the error between the two iterations is less than the threshold value; if it is reached, output the historical optimal solution, otherwise continue.

(iv) Check the connectivity of the network and repair the connectivity of the disconnected planning Figure based on the Boruvka algorithm. 
(v) Power flow calculation, calculation of line construction cost, network loss cost, and load shedding cost.

(vi) Transfer the planning Figure of the upper objective function to lower objective function, divide the planning Figures into groups of slow coherence, and calculate the weak connection coefficient of the system.

(vii) In the form of the penalty function, reflect the fitness value of the lower objective function to the upper objective function, and go to step (ii).

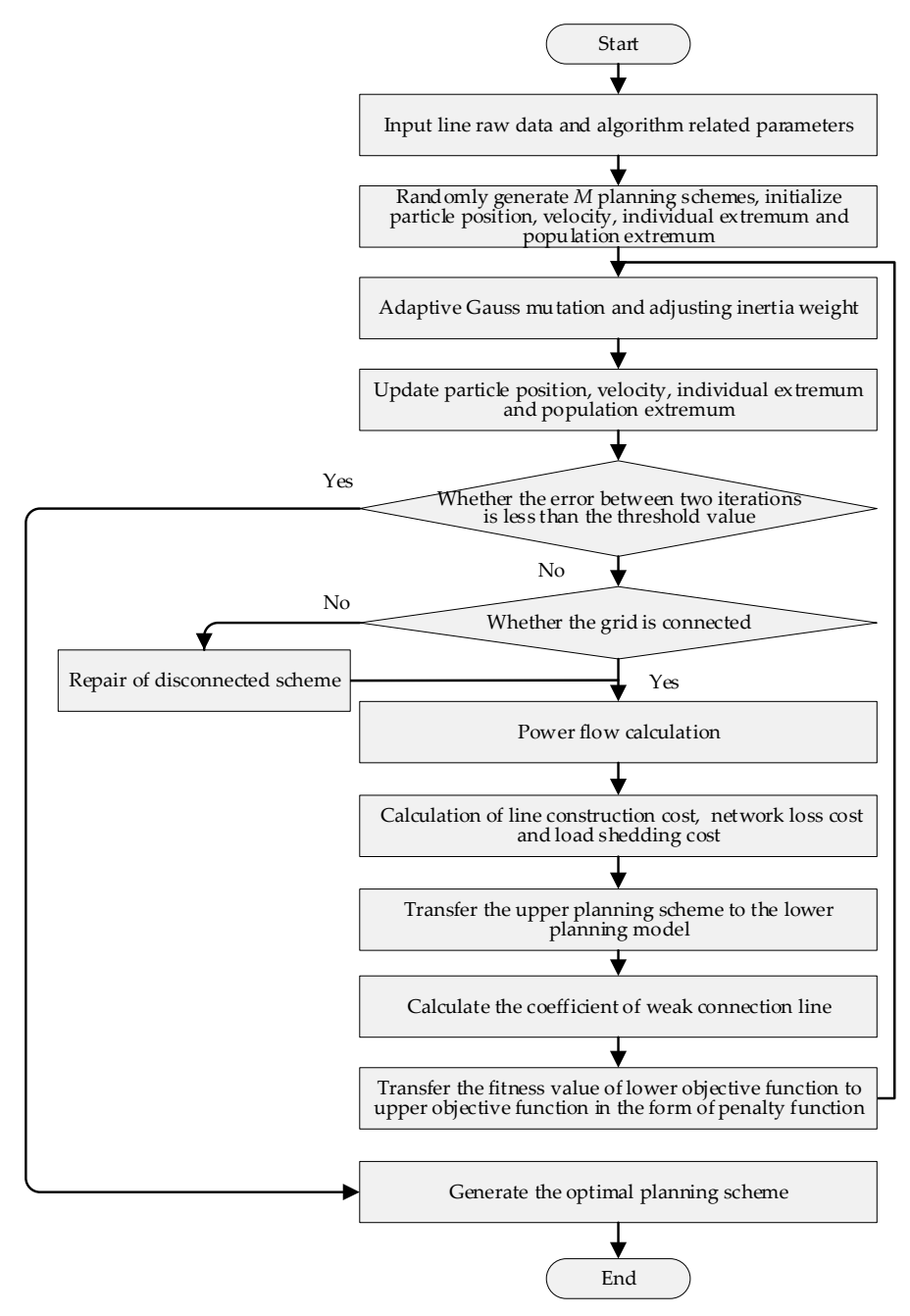

Figure 2. Flow chart for solving bi-level programming model of transmission network considering splitting control.

\section{Example Analysis}

In this paper, the 18 node IEEE Graver system is selected as the test example and the relevant parameter data reference of the test example, as shown in Tables 1 and 2. The parameters in the bi-level programming model are: assuming that all lines have, at most, three extendable lines; the power reference value is $100 \mathrm{MW}$; the line unit price is $\$ 71,338 / \mathrm{km}$; the fund discount rate is $10 \%$; the investment life is 15; the annual loss hours are $2000 \mathrm{~h}$; the unit price is $\$ 0.04 / \mathrm{kWh}$; the algorithm population is 60; the number of iterations is 200 , and the threshold is $1 \times 10^{-4}[25,26]$. 
Table 1. IEEE 18 node system network node parameters.

\begin{tabular}{ccc}
\hline Node & Generator Output (MW) & Load (MW) \\
\hline 1 & 0 & 55 \\
2 & 360 & 84 \\
3 & 0 & 154 \\
4 & 0 & 38 \\
5 & 760 & 639 \\
6 & 0 & 199 \\
7 & 0 & 213 \\
8 & 0 & 88 \\
9 & 0 & 259 \\
10 & 750 & 94 \\
11 & 540 & 700 \\
12 & 0 & 190 \\
13 & 0 & 110 \\
14 & 540 & 32 \\
15 & 0 & 200 \\
16 & 495 & 132 \\
17 & 0 & 400 \\
18 & 142 & 0 \\
\hline
\end{tabular}

Table 2. IEEE 18 node system network branch parameters.

\begin{tabular}{cccccccc}
\hline $\begin{array}{l}\text { Number } \\
\text { of Line }\end{array}$ & $\begin{array}{c}\text { Ends } \\
\text { Node }\end{array}$ & $\begin{array}{c}\text { AC } \\
\text { Resistance } \\
\text { (p.u.) }\end{array}$ & $\begin{array}{c}\text { AC } \\
\text { reactance } \\
\text { (p.u.) }\end{array}$ & $\begin{array}{c}\text { Length } \\
(\mathbf{k m})\end{array}$ & $\begin{array}{c}\text { Capacity } \\
\text { (MW) }\end{array}$ & $\begin{array}{c}\text { Number of } \\
\text { Existing Lines }\end{array}$ & $\begin{array}{c}\text { Number of Lines } \\
\text { That Can be Built }\end{array}$ \\
\hline 1 & $1-2$ & 0.001 & 0.0176 & 70 & 230 & 1 & 3 \\
2 & $1-11$ & 0.001 & 0.0102 & 40 & 230 & 0 & 3 \\
3 & $2-3$ & 0.001 & 0.0348 & 138 & 230 & 1 & 3 \\
4 & $3-4$ & 0.001 & 0.0404 & 155 & 230 & 1 & 3 \\
5 & $3-7$ & 0.001 & 0.0325 & 129 & 230 & 1 & 3 \\
6 & $4-7$ & 0.001 & 0.0501 & 200 & 230 & 0 & 3 \\
7 & $4-16$ & 0.001 & 0.0501 & 200 & 230 & 0 & 3 \\
8 & $5-6$ & 0.001 & 0.0267 & 106 & 230 & 1 & 3 \\
9 & $5-11$ & 0.001 & 0.0153 & 60 & 230 & 0 & 3 \\
10 & $5-12$ & 0.001 & 0.0102 & 40 & 230 & 0 & 3 \\
11 & $6-7$ & 0.001 & 0.0126 & 50 & 230 & 1 & 3 \\
12 & $6-13$ & 0.001 & 0.0126 & 50 & 230 & 0 & 3 \\
13 & $6-14$ & 0.001 & 0.0554 & 220 & 230 & 0 & 3 \\
14 & $7-8$ & 0.001 & 0.0151 & 60 & 230 & 1 & 3 \\
15 & $7-9$ & 0.001 & 0.0318 & 126 & 230 & 0 & 3 \\
16 & $7-13$ & 0.001 & 0.0126 & 50 & 230 & 0 & 3 \\
17 & $7-15$ & 0.001 & 0.0448 & 178 & 230 & 0 & 3 \\
18 & $8-9$ & 0.001 & 0.0102 & 40 & 230 & 1 & 3 \\
19 & $9-10$ & 0.001 & 0.0501 & 200 & 230 & 1 & 3 \\
20 & $9-16$ & 0.001 & 0.0501 & 200 & 230 & 0 & 3 \\
21 & $10-18$ & 0.001 & 0.0255 & 100 & 230 & 0 & 3 \\
22 & $11-12$ & 0.001 & 0.0126 & 50 & 230 & 0 & 3 \\
23 & $11-13$ & 0.001 & 0.0255 & 100 & 230 & 0 & 3 \\
24 & $12-13$ & 0.001 & 0.0153 & 60 & 230 & 0 & 3 \\
25 & $14-15$ & 0.001 & 0.0428 & 170 & 230 & 0 & 3 \\
26 & $16-17$ & 0.001 & 0.0153 & 60 & 230 & 0 & 3 \\
27 & $17-18$ & 0.001 & 0.0140 & 55 & 230 & 0 & 3 \\
\hline
\end{tabular}

\subsection{Planning Grid Analysis}

In this paper, a two-layer transmission network planning model considering splitting control is used for transmission network planning, and the results of the planning grid structure are compared with the results of transmission network planning considering the economy. The planning results of the two are shown in Figures 3 and 4, respectively, and the data comparison between the planning 
results of the two is shown in Table 3. The total investment cost in Table 3 includes lines of construction cost and network loss cost.

Comparison of the planning results of plan 1 and plan 2: 26 new lines have been built in plan 1, with a total investment cost of 26.2 million dollars, while 14 new lines have been built in plan 2, with a total investment cost of 15.7 million dollars. Compared with Figure 1, the total number of new lines and the total investment cost of Figure 2 are less. Although the economy of Figure 1 is slightly worse than that of Figure 2, the grid topology has more ring network structure, which improves the power supply reliability of the system. According to the data in Table 1, Figure 1 will hardly have line overload when operating under normal conditions and N-1 conditions, and it can operate more stably when the system is disturbed by the external environment, which can effectively reduce the line overload accident loss, and the overload loss of option 2 is much larger than that of option 1 . In the long run, it can make up the difference in investment cost between option 1 and option 2 . Moreover, as shown in Figure 3, the planned grid structure of Figure 1 can be divided into three areas: generators 2, 5 and 11 are in the same area, generators 10,16 and 18 are in the same area, generators 14 are in another area, and the number of weak connection lines between the areas is 6 , respectively lines $2-3$, 5-6, 4-16, 9-10, 9-16 and 12-13. The grid structure planned in Figure 2 is shown in Figure 4, which can be divided into four areas. Generators 2 and 11 are in the same area, generators 16 and 18 are in the same area, generators 10,14 and 5 are in the same area, respectively, and the number of weak connection lines between areas is 4 , which are lines $2-3,4-16,5-6$ and 9-16, respectively. Although Figure 1 has more weakly connected lines than Figure 2, the weak connection coefficient of the system is slightly smaller, and Figure 1 has fewer slow mode partitions and less potential oscillation modes. Overall, Figure 1 has better reliability than Figure 2.

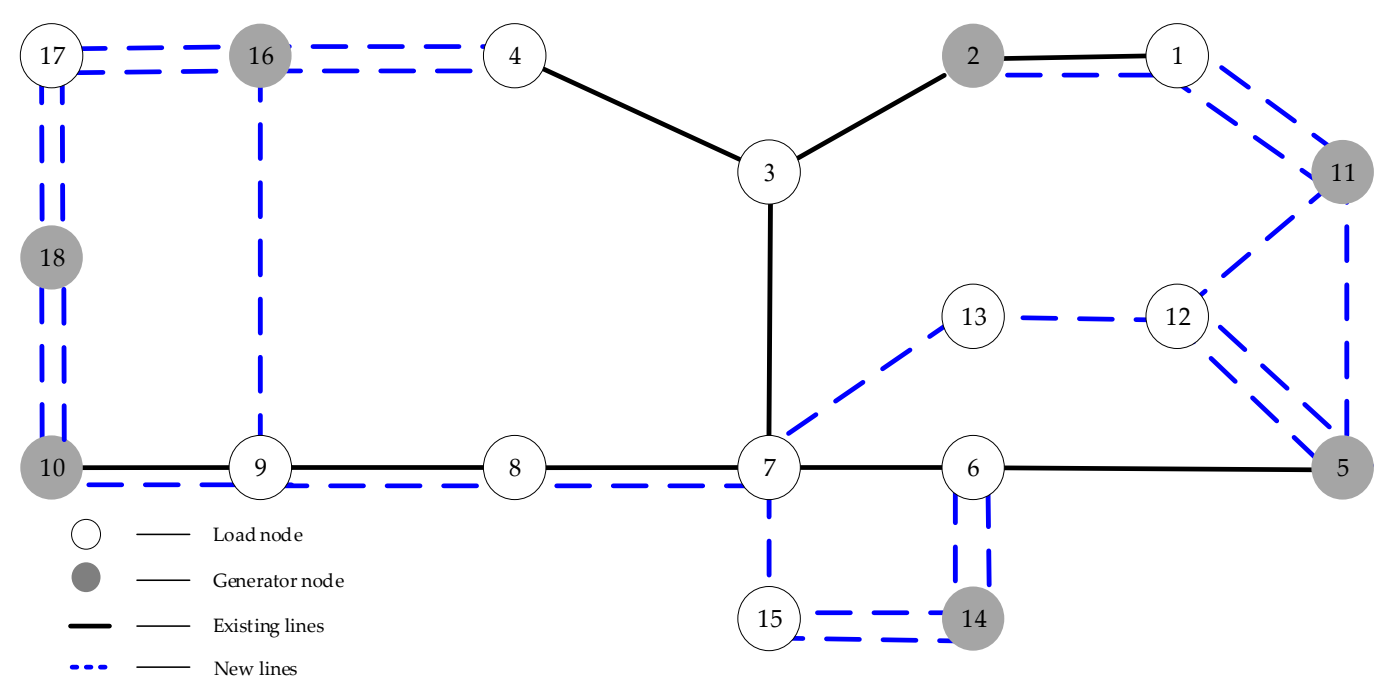

Figure 3. Planning results of the transmission network considering splitting control. 


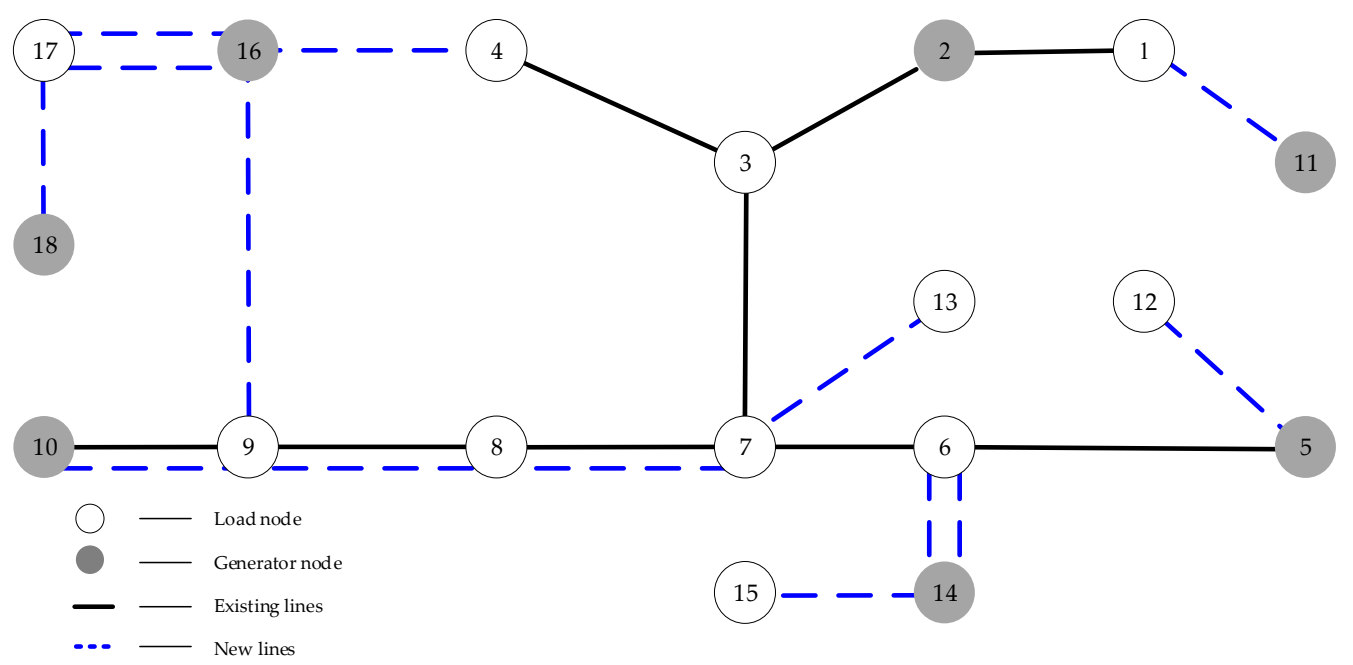

Figure 4. Planning results of the transmission network considering the economy.

Table 3. Comparison of transmission network planning results considering splitting control and economy.

\begin{tabular}{|c|c|c|}
\hline Planning Figure & $\begin{array}{c}\text { Figure 1: Transmission Network } \\
\text { Planning Results Considering } \\
\text { Splitting Control }\end{array}$ & $\begin{array}{c}\text { Figure 2: Transmission Network } \\
\text { Planning Results Considering } \\
\text { the Economy }\end{array}$ \\
\hline Number of new lines and circuits & $\begin{array}{c}1-2(1), 1-11(2) \\
4-16(2), 5-11(1) \\
5-12(2), 6-14(2) \\
7-8(1), 7-13(1) \\
7-15(1), 8-9(1) \\
9-10(1), 9-16(1) \\
10-18(2), 11-12(1) \\
12-13(1), 14-15(2) \\
16-17(2), 17-18(2)\end{array}$ & $\begin{array}{c}1-11(1), 4-16(1) \\
5-12(1), 6-14(2) \\
7-8(1), 8-9(1) \\
7-13(1), 9-10(1) \\
9-16(1), 14-15(1) \\
16-17(2), 17-18(1)\end{array}$ \\
\hline Total investment cost $/ 10^{6} \$$ & 26.2 & 15.7 \\
\hline Load shedding cost/10 $\$$ & 0 & 1.27 \\
\hline N-1 load shedding cost $/ 10^{4} \$$ & 0.06 & 88.67 \\
\hline Weak connection coefficient & 0.171 & 0.173 \\
\hline
\end{tabular}

\subsection{Feasibility Verification}

To verify the feasibility of Figure 1 splitting control in case of serious system failure, this paper simulates Figure 1 in PSD-BPA. The line fault is set as a three-phase short circuit fault, the fault start time is $0 \mathrm{~s}$, and the fault duration is $1 \mathrm{~s}$. The simulation adopts the generator model and the constant power load model, which take into account the change of magnetic flux, $E_{\mathrm{q}}{ }^{\prime}$ and $E_{\mathrm{d}}{ }^{\prime}$

\subsubsection{When there is a Fault at the Weak Connection Lines of the System}

A fault is set at the front end of the weak connection line $2-3$ of the planned grid structure in Figure 1, and the power angle curve of the generator after the fault is shown in Figure 5. As can be seen from Figure 5, when the fault occurs in line 2-3, the power angle of the generator in the system changes. Generators 2, 5, 11 is a group, generators 10,16, 18 is a group, and generators 14 is a group alone. According to the results of transmission network planning considering splitting control in Section 5.1, it can be seen that the weak connection lines 2-3, 5-6, 4-16, 9-10, 9-16, and 12-13 can be effectively separated from other clusters by splitting the selected splitting sets. According to the analysis of out-of- step splitting criteria based on the frequency of voltage bus, the actual out-of-step sections are lines $2-3,3-4,5-6,6-14,7-8,7-15,12-13,14-15$. It is proved that the feasibility of splitting 
the weak connection line, in case of failure at the weak connection lines, and the effectiveness of the model splitting control proposed in this paper.

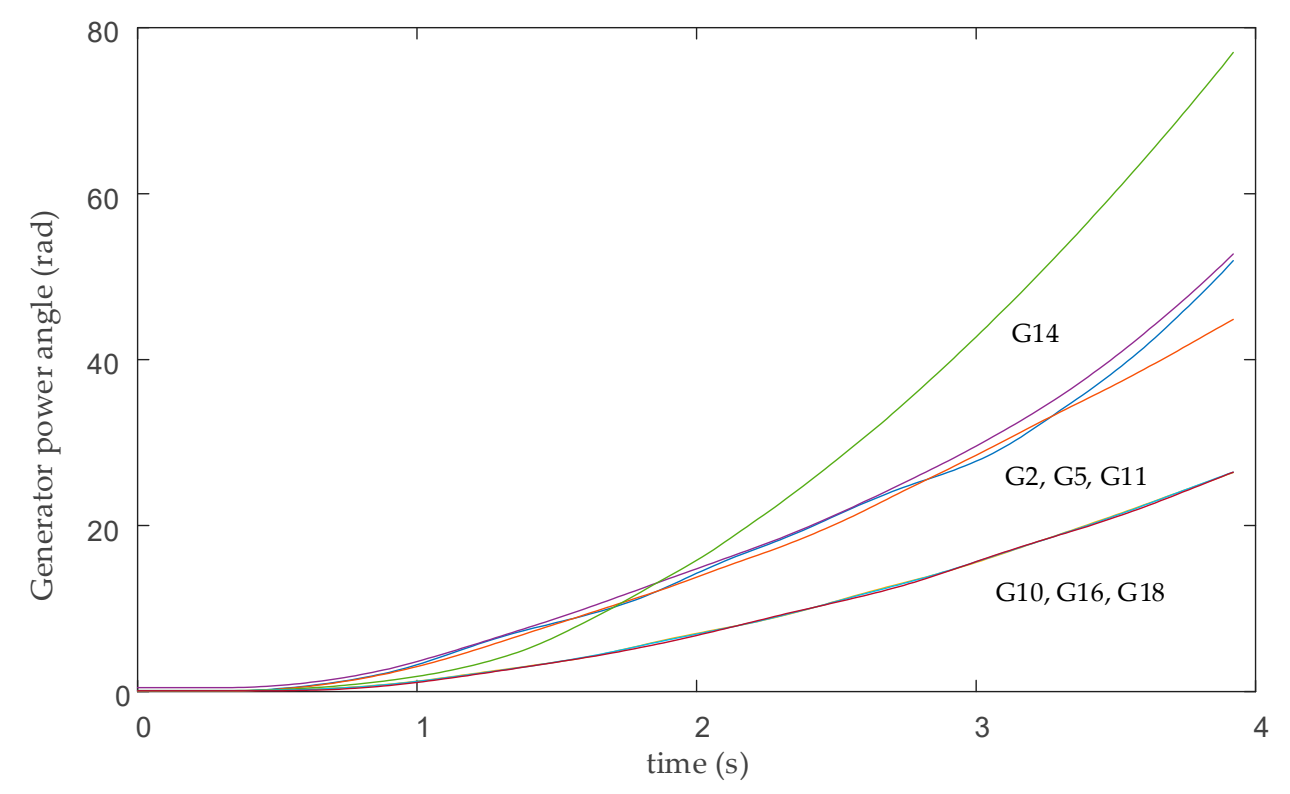

Figure 5. Generator power angle curve in case of line 2-3 fault.

\subsubsection{In Case of Internal Line Fault in Slow Mode Area}

In Figure 1, faults are set at lines 10-18 of the planned grid structure, and the power angle curve of the generator after faults is shown in Figure 6. As can be seen from Figure 6, when the line 10-18 fails, the system fleet is out-of-step. Generators 2, 5, 11 and 14 are a group, generators 16 and 18 are a group, and generators 10 is a separate group. According to the results of transmission network planning considering splitting control in Section 4.1, splitting at 4-16, 9-10 and 9-16 of the weak connection lines can effectively isolate out-of-step clusters from other clusters, to further find the most suitable splitting section to narrow the search scope and save search time. Through the out-of-step splitting criterion based on the frequency of voltage bus, it is found that the actual oscillation center migrates on lines 3-4, 4-16, 8-9, 9-10, 9-16, 10-18 and 16-17, which verifies the feasibility of taking the weak connection line as the lines to be selected for splitting in case of internal line fault in slow mode area and the effectiveness of the model splitting control proposed in this paper.

The above two sets of simulation verify the feasibility of taking the weak connection lines of the system as the lines to be selected for splitting and prove that the grid structure of the transmission network planning model proposed in this paper can cooperate with the splitting device to quickly partition the system in case of serious complex fault, avoid the spread of oscillation, reduce the search range and time of the optimal splitting section, and cooperate with the splitting control effectively carrying it out. 


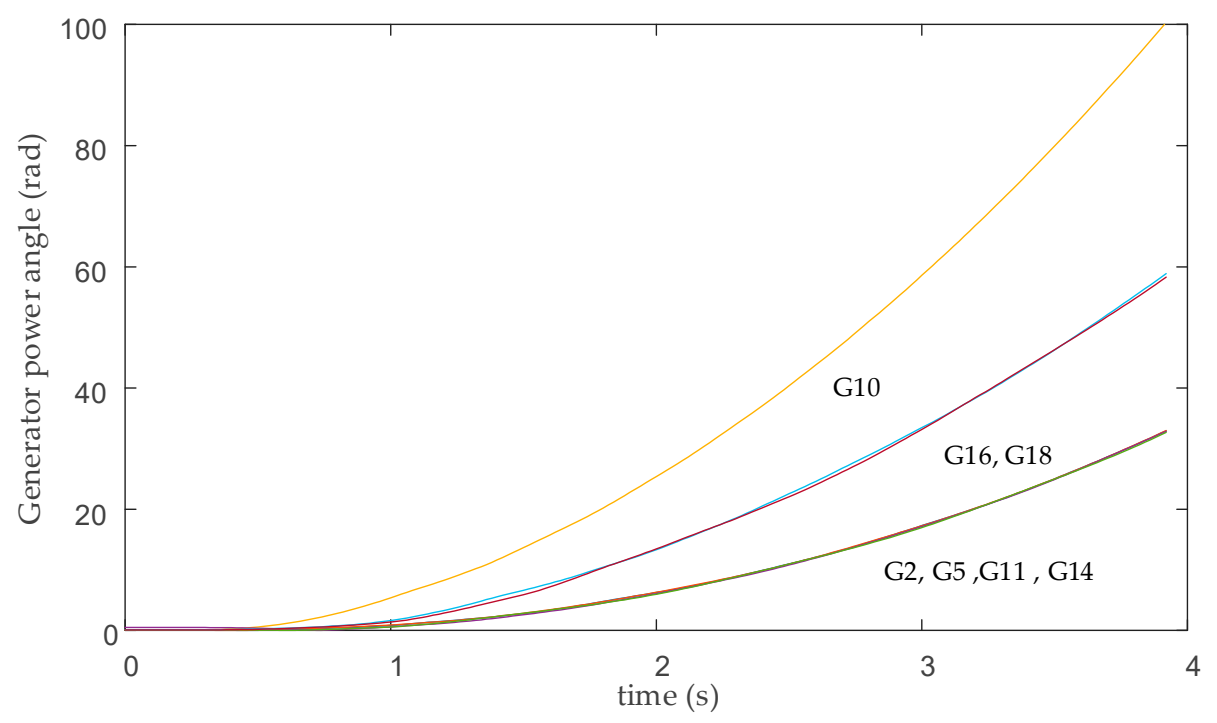

Figure 6. Generator power angle curve in case of line 10-18 fault.

\subsection{Fault Simulation Comparison}

To compare the effectiveness of Figures 1 and 2, the N-1 fault simulation of Figures 1 and 2 in PSD-BPA is carried out. The fault setting is the same as that in the feasibility verification. Select a group of fault simulation results for comparison, and take lines 6-14 as fault lines, as shown in Figures 7 and 8:

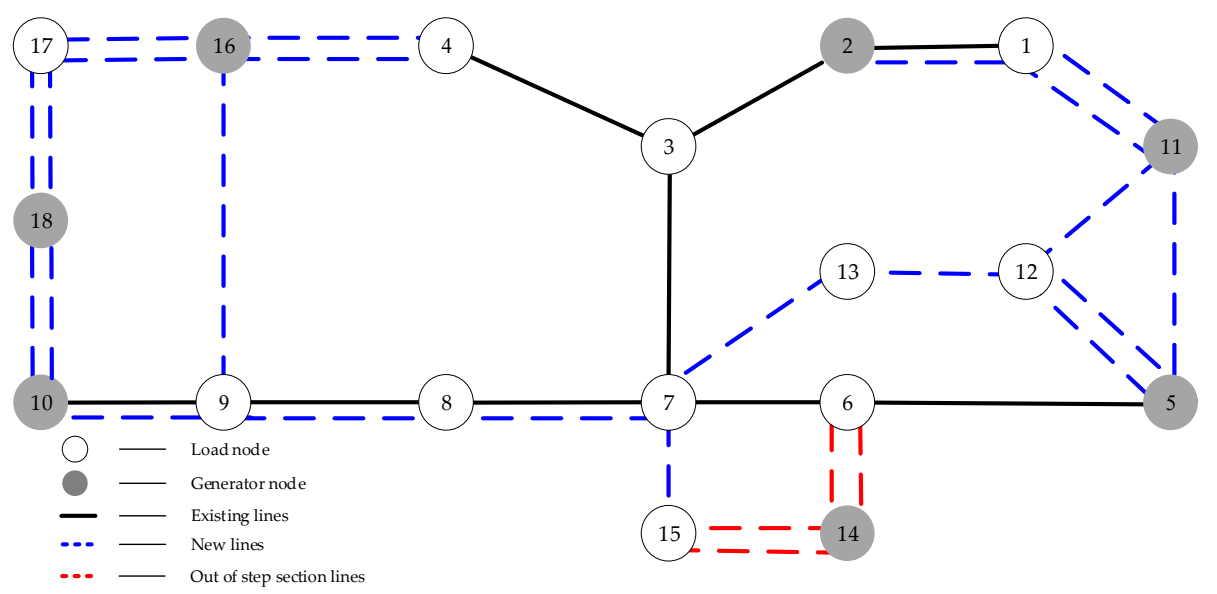

Figure 7. Out-of-step section of Figure 1 in case of line 6-14 failure.

As can be seen from Figures 7 and 8, the out-of-step section of Figure 1 is concentrated in the area where generators 14 is located; the out-of-step section of Figure 2 is distributed around generators 10 and generators 14. Compared with Figure 1, Figure 2 has a larger distribution area of out-of-step section and more complex oscillation mode when line 6-14 fails. Through the above fault analysis, it can be seen that the out-of-step section of Figure 1 is more concentrated in a certain area compared with Figure 2, which makes the splitting control more accurate and effective. 


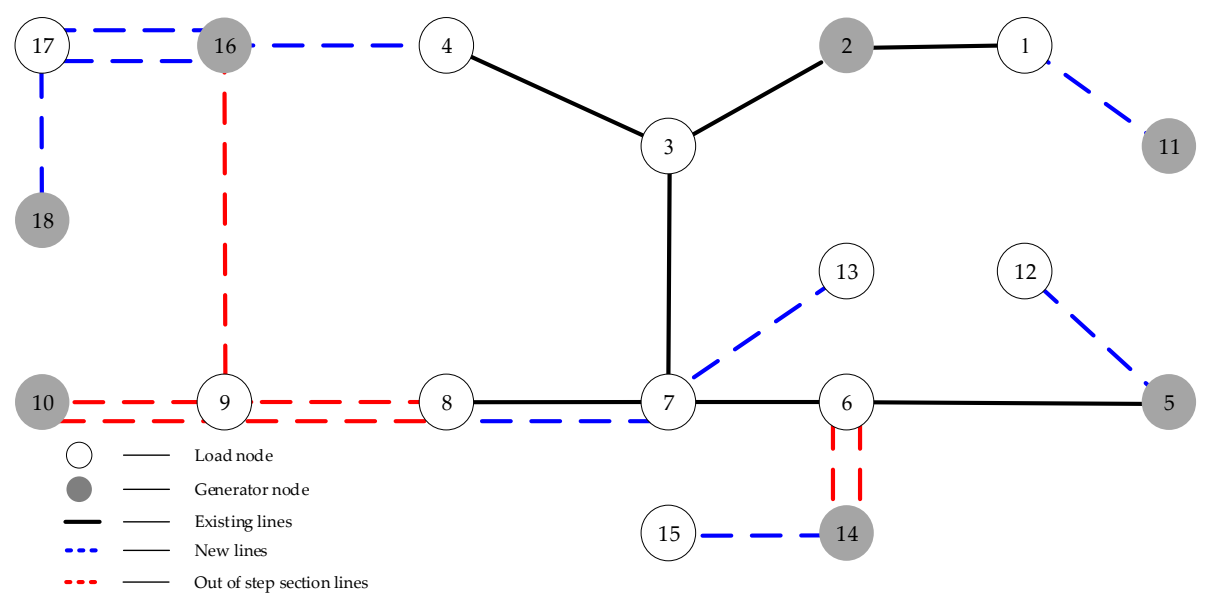

Figure 8. Out-of-step section of Figure 2 in case of line 6-14 failure.

\subsection{Simulation of Actual Power Grid}

An example of a power grid in East China in the summer of 2018 is used to verify the effectiveness of the proposed power grid planning model. The calculation example includes 812 nodes, 62 generator nodes, and the total load of the system in a certain period is $32,432 \mathrm{MW}$. The schematic diagram of the power grid transmission line is shown in Figure 9, which can be roughly divided into 14 areas such as XH, HD, SJ, XY, NQ, TW, LT, YD, SL, JA, YG, GL, YH, WE, etc. Based on the transmission network planning model considering splitting control, the planning is carried out based on lines above $500 \mathrm{kV}$ in the grid, and the planned new lines are shown in Table 4.

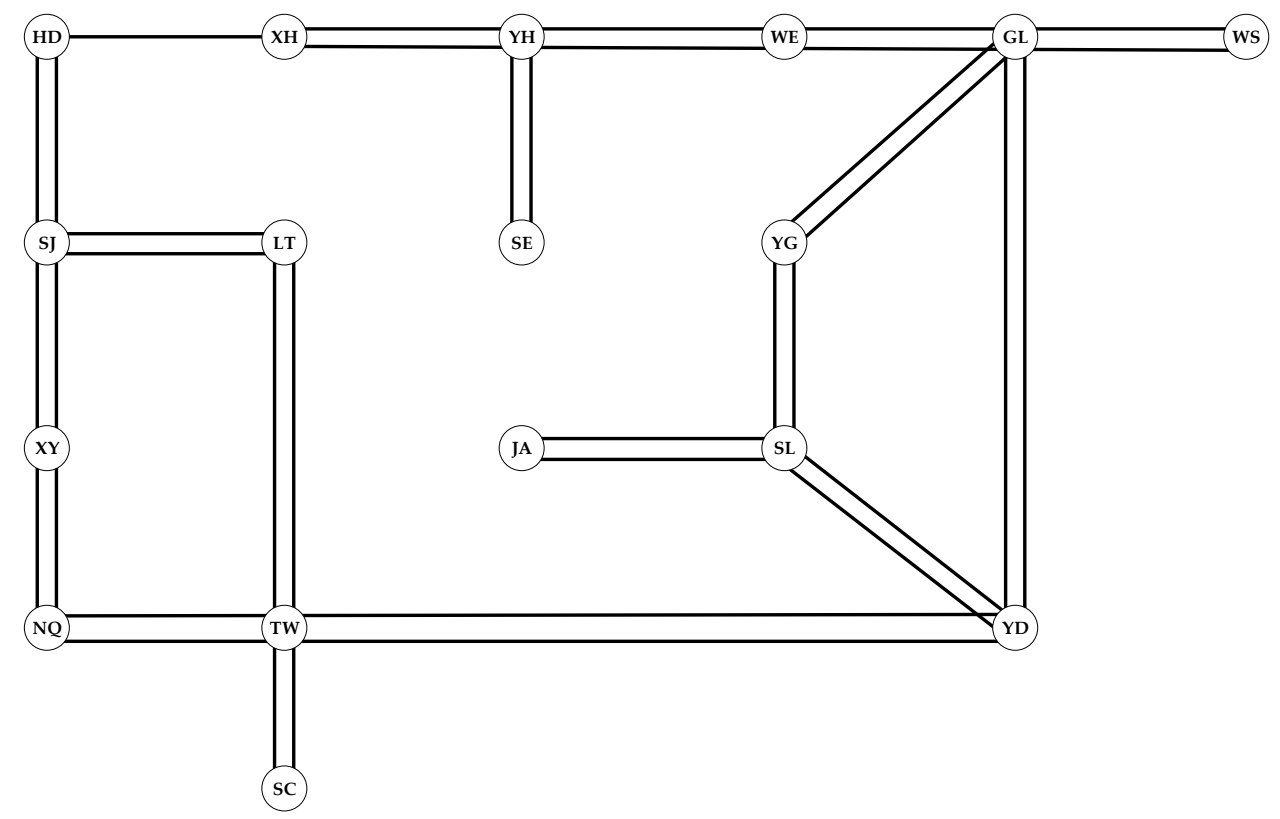

Figure 9. Schematic diagram of the transmission line for a power grid in East China.

Table 4. Planning new lines.

\begin{tabular}{cc}
\hline Figure & Number of New Lines and Circuits \\
\hline $\begin{array}{c}\text { Transmission network planning results considering } \\
\text { splitting control }\end{array}$ & HD-SJ (1), HD-XH (1), SJ-XY (1) \\
\hline
\end{tabular}

According to the simulation analysis, the initial grid can be divided into four slow mode areas, with $\mathrm{LT}$ and TW as one area, HD, SJ, XY and NQ as one area, and the rest as one area; the weak 
connection lines of the initial grid are HD-XH, SJ-LT, NQ-TW, LT-TW, and TW-YD respectively. The optimized transmission network planning model considering splitting control is divided into two slow mode areas; TW is one area, and the rest is one area; the weak connection lines of the optimized grid are NQ-TW, LT-TW, and TW-YD. Compared with the original network, the optimized network has less slow mode partition and less weak connection lines.

To verify the effectiveness of the proposed transmission network planning model considering splitting control in the actual grid, three-phase short-circuit fault is set at the GL-YD line of the planning grid, and the initial grid, $0.1 \mathrm{~s}$ fault starts, $0.8 \mathrm{~s}$ fault duration, and the generator power angle curve is shown in Figures 10 and 11. It can be seen from Figures 10 and 11 that after the initial grid fault, the generators are divided into 4 groups: GL and WE are a group, YD is a group, TW is a group, and the rest of the areas are a group; however, after the optimization of this paper, the generators are divided into 3 groups, among which GL and YD are each a group and other areas are a group. Compared with the optimized grid structure, the initial grid structure has more fault clusters, more complex oscillation modes, and larger distribution areas of out-of-step sections, which can verify the effectiveness of the proposed transmission network planning model considering splitting control in the actual grid.

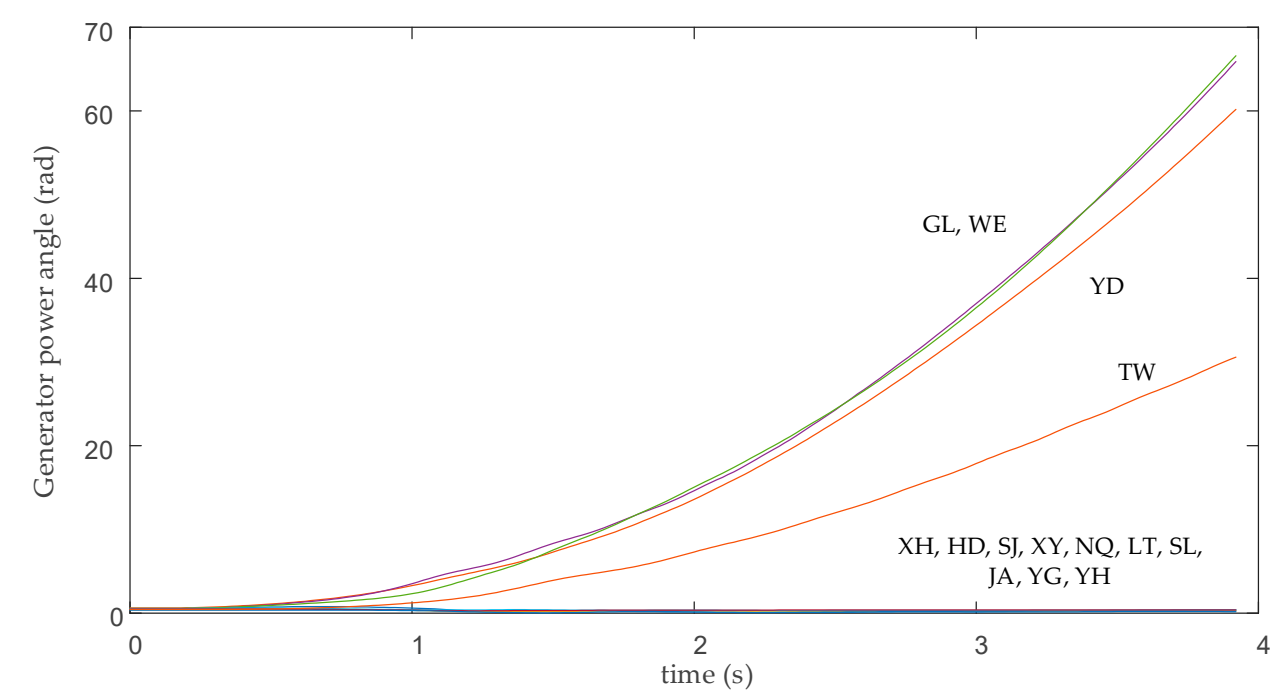

Figure 10. Power angle curve of the generator after line GL-YD fault of the initial grid structure.

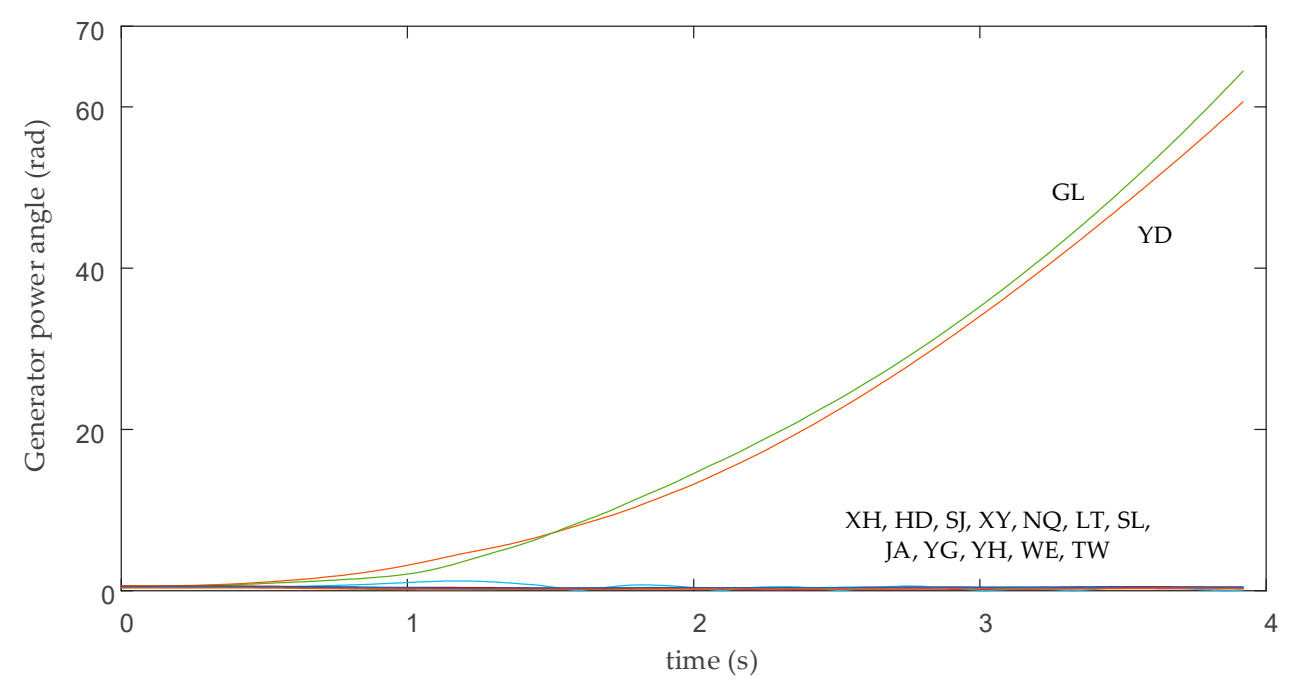

Figure 11. Power angle curve of the generator after line GL-YD fault of the optimized grid structure. 


\section{Conclusions}

To deal with the increased risk of out-of-step oscillations between regions and improve the ability of interconnected power grid to resist failure risk, this paper proposes a two-level planning model of transmission network considering splitting control, and obtains the following conclusions:

- Based on the modified cosine factor and graph theory, this paper proposes an improved method of identifying and screening the weak connection lines of the system, which can screen the weak connection lines more accurately and automatically, and does not rely on the judgment of the network topology.

- Compared with the traditional transmission network planning, this paper proposes the concept of weak connection coefficient of the system and establishes the double-layer planning model of the transmission network with the minimum weak connection coefficient as the optimization goal. The model can optimize the system partition, reduce the decision-making space of the splitting lines, and cooperate with the splitting control rapidly.

- In this paper, a hybrid algorithm based on Boruvka algorithm and particle swarm optimization algorithm with adaptive mutation and inertia weight is used to solve the IEEE 18 node standard calculation example and a real power grid in East China. Through PSD-BPA fault simulation of the planning results, the feasibility of the weak connection lines as the lines to be selected is verified, and the adaptability of the power grid operation state change is verified. Furthermore, the transmission network planning model proposed in this paper can better cooperate with the splitting control.

Author Contributions: The authors confirm their contributions to the paper as follows: F.T. and C.X. proposed the idea and wrote the paper; X.G. and Y.Z. revised the manuscript; N.D. and B.H. reviewed the results and approved the final version of the manuscript. All authors have read and agreed to the published version of the manuscript.

Funding: This study was funded by the National Natural Science Foundation of China (grant number 51977157).

Acknowledgments: We sincerely thank the editor and the anonymous reviewers of the paper for their kind support. We also want to thank the Grid Corporation for providing relevant information and data.

Conflicts of Interest: The authors declare no conflict of interest.

\section{Nomenclature}

\section{Abbreviations}

IEEE

PSD-BPA

$\mathrm{N}, \mathrm{N}-1, \mathrm{~N}-2, \mathrm{~N}-\mathrm{K}$

PSO

MW

AC

p. u.

XH, HD, SJ, XY, NQ, TW, LT, YD,

SL, JA, YG, GL, YH, WE

G2, G5, G10, G11, G14, G16, G18
Institute of Electrical and Electronics Engineers

Power System Department-Bonneville Power

Administration

any $\mathrm{K}(0,1, \ldots, \mathrm{K})$ independent components of the $\mathrm{N}$

components of the power system are removed after

failure, without damaging the stability of the system.

Particle Swarm Optimization

megawatt

Alternating Current

per unit

place name

generator number 


\section{Parameters}

A

$n$

$\Omega$

$\delta_{\mathrm{i}}$

$\omega_{\mathrm{i}}$

$H_{\mathrm{i}}$

$D_{\mathrm{i}}$

$P_{\text {mi }}$

$P_{\text {ei }}$

$E_{\mathrm{i}}, E_{\mathrm{j}}$

$\delta_{\mathrm{ij}}$

$G_{i j}$

$B_{\mathrm{ij}}$

$Y_{\mathrm{ij}}$

$P_{\text {ei0 }}$

$\delta_{\mathrm{i} 0}$

K

$L_{\mathrm{ij}}$

$D_{\mu_{m}, \min }^{l}$

$D_{\mu_{m}, \max }^{\mu_{m}, \min }$

a

$r$

$k_{1}$

$L_{\mathrm{i}}$

$m_{1}$

$\tau_{\mathrm{i}}$

$k_{2}$

$r_{\mathrm{i}}$

$U_{\mathrm{i}}$

$k_{3}$

$V_{\mathrm{i}}, V_{\mathrm{j}}$

$\theta_{\mathrm{ij}}$

$P_{\mathrm{G}, \mathrm{i}}, Q_{\mathrm{G}, \mathrm{i}}$

$P_{\mathrm{L}, \mathrm{i}}, Q_{\mathrm{L}, \mathrm{i}}$

$P_{\text {ijmax }}$

$P_{\mathrm{G}}$

$P_{\mathrm{G}, \min }, P_{\mathrm{G}, \max }$

$l_{\text {imax }}$

$\omega$

d

$c_{1}, c_{2}$

$r_{1}, \mathrm{r}_{2}$

$p_{\text {id }}$

$p_{\text {gd }}$

$p$

$\eta$

$\mu$

$\omega_{\text {start }}, \omega_{\text {end }}$

$t_{\max }$

K

$E_{\mathrm{q}}{ }^{\prime}, E_{\mathrm{d}}{ }^{\prime}$ state matrix

number of generator sets

rated frequency

rotor angle of generator $i$

speed of the generator $i$

the inertia time constant of the generator $i$

damping constant

the mechanical input power of the generator $i$

the electromagnetic output of the generator $i$

potential vector amplitude of the generator $i$ and $j$

considering transient reactance

power angle difference between the generator $i$ and $j$

the real part of the reduced node admittance matrix

of the system

the imaginary part of the reduced node admittance

matrix of the system

reduced node admittance matrix of the system

generator power steady-state value

generator power angle steady-state value

system state matrix

clustering matrix

the lower limit of line sensitivity

the upper limit of line sensitivity

fund discount rate

construction years

line construction cost per unit length

length of line $i$

line set

annual loss hours of electric energy of line $i$

unit electricity price

the resistance of line $i$

the voltage of line $i$

unit load cut-off compensation cost

the voltage of node $i$ and $j$

phase angle difference between node $i$ and node $j$

active power output and reactive power output of

node $i$

active load and a reactive load of node $i$

maximum line capacity

generator power output

minimum and maximum generator power output

maximum number of lines

inertia weight

current particle

learning factor

random variable between $[0,1]$

individual extremum

population extremum

mutation threshold

random variables obeying $[0,1]$ Gaussian distribution random variable between $[0,1]$

the initial and final inertia weight

maximum number of iterations

constant

transient direct axis and quadrature axis

electromotive force 


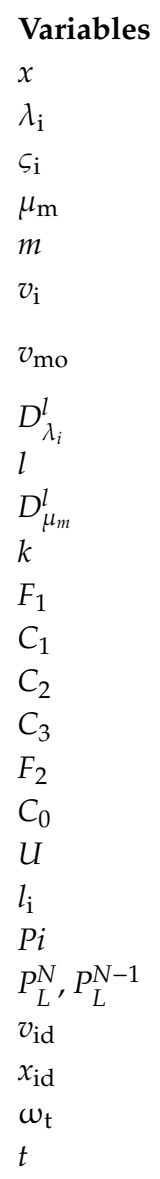

\author{
system state variable \\ eigenvalues of the system state matrix \\ eigenvalues of second-order systems \\ a set of eigenvalues for the system state matrix \\ number of slow mode eigenvalues \\ characteristic variables of slow mode eigenvalues \\ mean value of a characteristic variable of slow mode \\ eigenvalue \\ the sensitivity of slow mode eigenvalues $\lambda_{\mathrm{i}}$ to line $l$ \\ transmission line \\ the sensitivity of slow mode eigenvalue group to line $l$ \\ number of weak connection lines \\ upper objective function \\ line construction cost \\ network loss cost \\ load shedding cost \\ lower-level objective function \\ weak connection coefficient of the system \\ penalty value for not meeting the lower constraint \\ number of new circuits of line $i$ \\ active power transmitted by line $\mathrm{i}$ \\ removal load under $\mathrm{N}$ and $\mathrm{N}-1$ conditions \\ particle velocity \\ particle position \\ current inertia weight \\ iteration times
}

\section{References}

1. Xiao, C.F.; Tang, F.; Liu, D.C. Power Transmission Network Expansion Planning Research Considering System Weak Connection Recognition. In Proceedings of the 2019 IEEE Innovative Smart Grid Technologies-Asia (ISGT Asia), Chengdu, China, 21-24 May 2019; pp. 191-195.

2. Wu, W.P.; Hu, Z.C. Transmission Network Expansion Planning Based on Chronological Evaluation Considering Wind Power Uncertainties. IEEE Trans. Power Syst. 2018, 33, 4787-4796. [CrossRef]

3. Zhou, Y.; Zhou, Q.M.; Yu, H.Q. Splitting control decision Figure design and splitting control analysis in a provincial power grid. In Proceedings of the 2016 12th World Congress on Intelligent Control and Automation (WCICA), Guilin, China, 12-15 June 2016; pp. 2338-2342.

4. Tang, F.; Yang, J. Out-of-step oscillation splitting criterion based on bus voltage frequency. J. Mod. Power Syst. Clean Energy 2015, 3, 341-352. [CrossRef]

5. Liu, Y.Y. Analysis of Brazilian blackout on 21st March 2018, and revelations to security for human grid. In Proceedings of the 2019 4th International Conference on Intelligent Green Building and Smart Grid (IGBSG), Yichang, China, 6-9 September 2019; pp. 1-5.

6. Licia, P.; Carlos, R.M. The Venezuelan energy crisis: Renewable energies in the transition towards sustainability. Renew. Sustain. Energy Rev. 2019, 105, 415-426.

7. Zhang, S.; Zhang, Y.X. A Novel Out-of-Step Splitting Protection Based on the Wide Area Information. IEEE Trans. Smart Grid 2017, 8, 41-51. [CrossRef]

8. Zhang, S.; Zhang, Y.X. Characteristic Analysis and Calculation of Frequencies of Voltages in Out-of-Step Oscillation Power System and a Frequency-Based Out-of-Step Protection. IEEE Trans. Power Syst. 2019, 34, 205-214. [CrossRef]

9. Stott, B.; Marinho, J.L. Linear programming for power-system network security applications. IEEE Trans. Power App. Syst. 1979, 98, 837-848. [CrossRef]

10. Street, A.; Oliveira, F. Contingency-constrained unit commitment with n-k security criterion: A robust optimization approach. IEEE Trans. Power Syst. 2011, 26, 1581-1590. [CrossRef] 
11. Reddy, S.S. Multi-Objective Based Congestion Management Using Generation Rescheduling and Load Shedding. IEEE Trans. Power Syst. 2017, 32, 852-863.

12. Kroposki, B.; Sen, P.K. Optimum sizing and placement of distributed and renewable energy sources in electric power distribution systems. IEEE Trans. Ind. Apps. 2013, 49, 2741-2752. [CrossRef]

13. Sajad, N.R.; Nazanin, J. Smart distribution grid multistage expansion planning under load forecasting uncertainty. IET Gener. Transm. Distrib. 2016, 10, 1136-1144.

14. Li, Z.H.; Lin, Y.; Chen, W.H. Fuzzy comprehensive evaluation for transmission grid planning based on combination weight. In Proceedings of the International Conference on Power and Energy, CPE 2014, Shanghai, China, 29-30 November 2014; pp. 91-96.

15. Yorino, N.; Abdillah, M. Robust Power System Security Assessment Under Uncertainties Using Bi-Level Optimization. IEEE Trans. Power Syst. 2017, 33, 352-362. [CrossRef]

16. Tan, K.M.; Ramachandaramurthy, V.K. Optimal vehicle to grid planning and scheduling using double layer multi-objective algorithm. Energy 2016, 112, 1060-1073. [CrossRef]

17. Ni, J.M.; Shen, C. An on-line weak-connection identification method for controlled islanding of power system. Proc. Chinese Soc. Electr. Eng. 2011, 31, 24-30.

18. Yang, Y.G.; Duan, Q.G.; Wu, G.B. Slow coherency based adaptive controlled islanding Figure of the China Southern Power Grid. In Proceedings of the 2015 IEEE PES Asia-Pacific Power and Energy Engineering Conference (APPEEC), Brisbane, Australia, 15-18 November 2015; pp. 1-5.

19. Li, M.X. An improved fcm clustering algorithm based on cosine similarity. In Proceedings of the 2019 International Conference on Data Mining and Machine Learning, ICDMML 2019, Hong Kong, China, 28-30 April 2019; pp. 103-109.

20. Ma, Z.Y.; Liu, F. Fast Searching Strategy for Critical Cascading Paths Toward Blackouts. IEEE Access 2018, 6, 36874-36886. [CrossRef]

21. Alinezhad, B.; Karegar, H.K. Out-of-Step Protection Based on Equal Area Criterion. IEEE Trans. Power Syst. 2017, 32, 968-977. [CrossRef]

22. Song, H.L.; Wu, J.Y. A wide-area measurement systems-based adaptive strategy for controlled islanding in bulk power systems. Energies 2014, 7, 2631-2657. [CrossRef]

23. Kokotovic, P.V.; Avramovic, B. Coherency based decomposition and aggregation. Automatica 1982, 18, 47-56. [CrossRef]

24. Chow, J.H.; Kokotovic, P.V. Decomposition of near-optimum regulators for systems with slow and fast modes. IEEE Trans. Autom. Control 1976, 21, 701-705. [CrossRef]

25. Yang, B.; Vittal, V. Slow-Coherency-Based Controlled Islanding-A Demonstration of the Approach on the August 14, 2003 Blackout Scenario. IEEE Trans. Power Syst. 2006, 21, 1840-1847. [CrossRef]

26. Wang, X.M.; Vittal, V. Tracing generator coherency indices using the continuation method: A novel approach. IEEE Trans. Power Syst. 2005, 20, 1510-1518. [CrossRef]

27. Ni, J.M.; Shen, C. Adaptive islanding control based on slow coherency-Part II: Practical area partition method. Proc. Chinese Soc. Electr. Eng. 2014, 34, 4865-4875.

28. Xu, G.Y.; Vittal, V. Slow Coherency Based Cutset Determination Algorithm for Large Power Systems. IEEE Trans. Power Syst. 2010, 25, 877-884. [CrossRef]

29. Seo, J.H.; Im, C.H. Multimodal function optimization based on particle swarm optimization. IEEE Trans. Magn. 2006, 42, 1095-1098. [CrossRef]

30. Zhang, J.T.; Xia, P.Q. An improved PSO algorithm for parameter identification of nonlinear dynamic hysteretic models. J. Sound Vibr. 2017, 389, 153-167. [CrossRef]

(C) 2020 by the authors. Licensee MDPI, Basel, Switzerland. This article is an open access article distributed under the terms and conditions of the Creative Commons Attribution (CC BY) license (http://creativecommons.org/licenses/by/4.0/). 